\title{
LE PROBLĖME. DE LA MESURE DES COURANTS MARINS
}

\author{
THE PROBLEM OF SPEED MEASUREMENTS \\ OF MARINE CURRENTS \\ English Synopsis p. 119. \\ par $V$. ROMANOVSKY, \\ Ingénieur-Docteur \\ Professeur d'Océanographie à l'O, R. S. C.
}

Que ce soit par le jeu des marées, des courants ou de la houle, les eaux des mers sont en perpétuel mouvement. En général celui-ci est particulièrement violent dans les couches superficielles, il s'atténue ensuite progressivement à mesure que l'on descend en profondeur et, dans les grands fonds, il est à peine perceptible.

Si la houle (d'après Gerstner) déplace les particules fluides, elle ne leur imprime qu'un mouvement orbitaire. Les déplacements moyens sont nuls au large. En eau profonde, les particules fluides restent sur place; ainsi un corps flottant placé à la surface, monte et descend sous l'influence de la houle sans être affecté d'aucun mouvement global.

\section{CLASSIFICATION DES COURANTS}

Les courants marins qui sont la conséquence des déplacements horizontaux des particules fluides peuvent avoir plusieurs origines. Suivant qu'ils sont apériodiques ou périodiques, on peut les classer en deux grands groupes:

\section{$1^{\circ}$ Courants apériodiques.}

Ils riont aucune périodicité dans leurs variations. Parmi ces courants on peut distinguer :

a) Les courants de densité. Ils sont liés aux différences de température et de salinité (donc de densité) existant, le long de verticales, entre deux points de l'Océan. A cette catégorie, appartiennent les grands courants océaniques tels que le Gulf Stream, le Kuroshio ou le courant Equatorial. Ces courants transportent de très grandes quantités d'eau. La rotation de la Terre influe sur leur trajectoire théorique, la déviant vers la droite dans l'hémisphère boréal et vers la gauche dans I'hémisphère austral (fig. 1). Leur extension en profondeur est souvent considérable; elle peut atteindre plusieurs centaines de mètres pour les grands courants. Ils sont actuellement assez bien connus par des mesures directes en surface, par des calculs pour les couches profondes. b) Les courants de dérive. Ils sont dus au vent, lorsque celui-ci souffle pendant un certain temps dans une direction constante. Ils se produit alors un entraînement superficiel des particules fluides et, si la Terre était immobile, le courant, ayant ainsi pris naissance, porterait dans la direction dans laquelle souffle le vent. Mais la rotation de la Terre provoque une déviation du courant qui, en mer profonde, est inclinée de $45^{\circ}$ vers la droite dans l'hémisphère Nord et

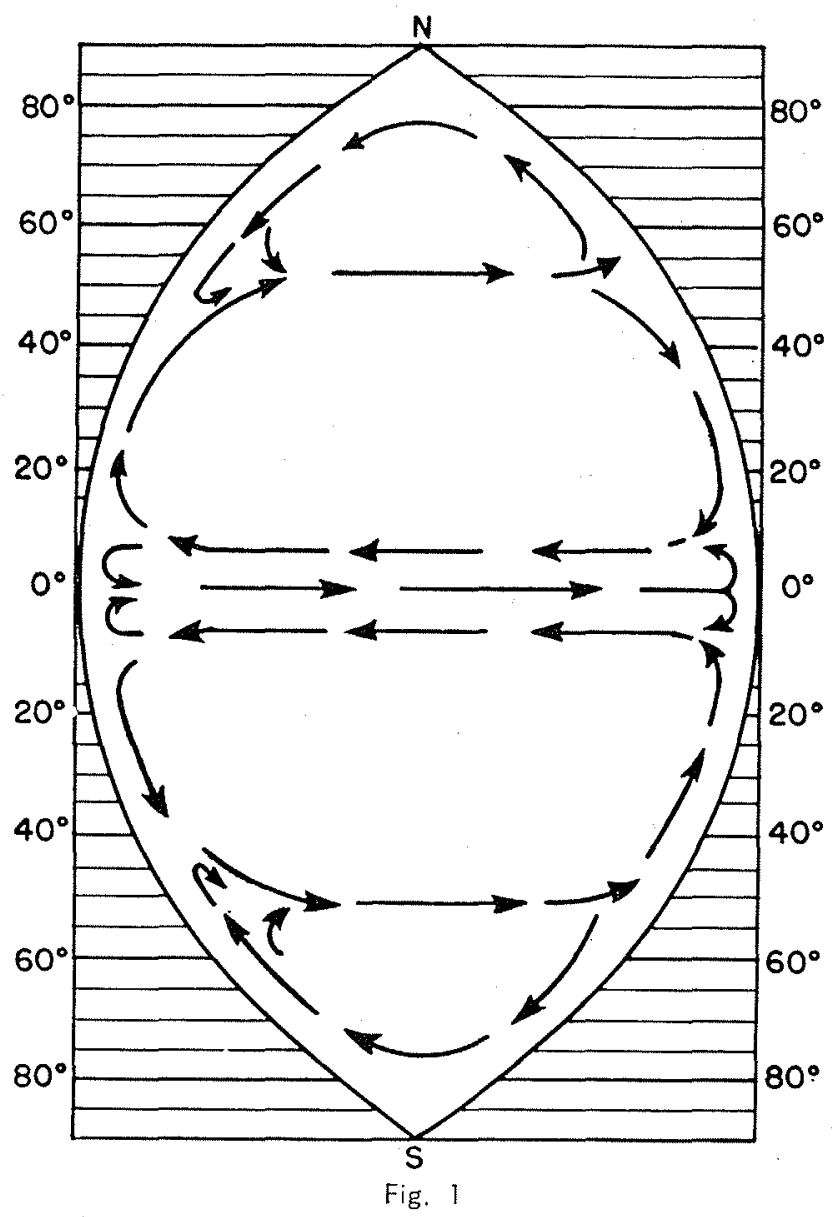

Réportition schématique des courants superficiels dans les deux hémisphères. 
vers la gauche dans l'hémisphère Sud. La vitesse du courant de dérive diminue avec la profondeur suivant une loi exponentielle, pendant que l'angle avec la direction du vent augmente.

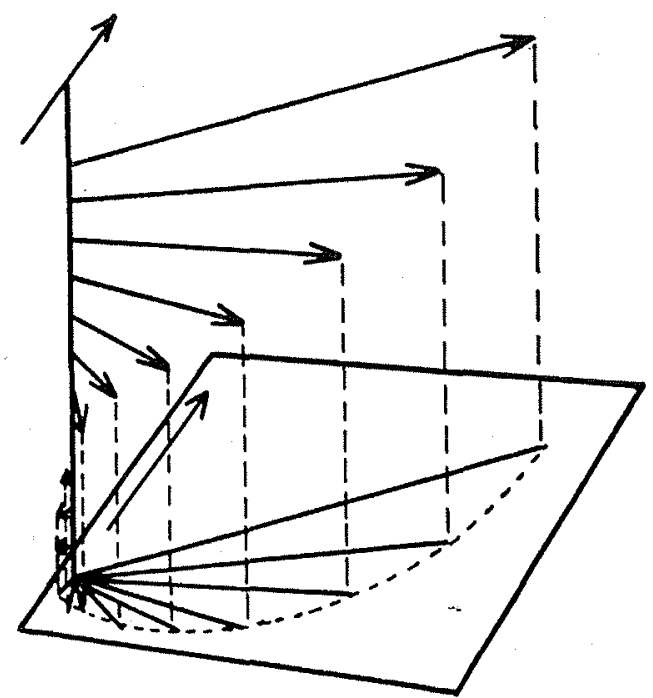

Fig. 2

Représentation schématique d'un courant de dérive an eau profonde, montrant les variations de la vitesse et de la direction avec la profondeur (spirale d'Ekman).

Le schéma de la figure 2 donne, en fonction de la profondeur, les variations de la vitesse et de la direction d'un courant de dérive dû au vent.

Dans les régions tempérées, la vitesse d'un courant de dérive peut atteindre une valeur égale à $1,5 \%$ de la vitesse du vent. Ainsi pour un vent de $40 \mathrm{~km} . / \mathrm{h}$. on peut avoir un courant marin de $15 \mathrm{~cm} . / \mathrm{sec}$, donc sensiblement d'un tiers de nœud.

Autour du continent antartique, un anneau de mers est soumis à des vents persistants et violents qui soufflent d'ouest en est. Ces mouvements de l'atmosphère provoquent une circulation océanique superficielle qui fait tourner inlassablement les eaux antarctiques d'ouest en est, mais les courants de dérive, ainsi créés, sont déviés vers la gauche par rapport à la direction des vents. D'après certains observateurs des expéditions polaires, cette déviation est en moyenne de $34^{\circ}$ et non de $45^{\circ}$ comme le prévoit la théorie. Cette anomalie a été expliquée comme étant due à un mouvement de l'eau directement sous la glace et à la résistance offerte au vent par la masse du pack-ice. Le vent et les courants des mers antarctiques transportent la banquise qui tourne éternellement autour du continent bloquant telle région, ou débloquant telle autre et ne permettant aucune prévision sur leur extension ou leur position. Les courants superficiels des mers antarctiques fournissent un grandiose exemple des courants de dérive dus au vent. c) Les courants de pente. Lorsqu'un courant de dérive a amené un afflux d'eau sur une côte, le niveau de la surface des eaux s'élève et quand le vent cesse de souffler, les eaux accumulées s'écoulent. Par fonds importants, la direction de l'écoulement fait un angle de $90^{\circ}$ avec la direction de la pente descendante. Celle-ci étant nécessairement perpendiculaire au littoral, les eaux s'écouleront parallèlement au rivage dès qu'elles auront atteint des profondeurs suffisantes.

Les courants de dérive et de pente peuvent s'ajouter et, dans ce cas, la vitesse du courant peut atteindre $3,5 \%$ de la vitesse du vent. Ainsi un courant de dérive dû à un vent de 40 $\mathrm{km}$./h., associé à un courant de pente, peut donner un courant résultant de près de 0,7 nœud.

\section{$2^{\circ}$ Courants périodiques.}

IIs sont dotés d'une périodicité et changent de sens à des intervalles de temps bien définis. Parmi ces courants on peut distinguer :

a) Les courants de marée. Ils ont la même période que les oscillations de marée. En certaines régions, où l'amplitude de la marée est importante, ils peuvent être très violents et atteindre plusieurs nouds. Dans les étranglements, ou au passage de seuils sous-marins, leur vitesse peut également atteindre des valeurs élevées.

Dans une onde progressive, le flot porte dans la direction de propagation et le jusant en sens inverse, les étales ont lieu d̀ l'instant du passage au niveau moyen. Dans une onde stationnaire, le flot est le courant qui accompagne la montée des eaux, le jusant, la baisse. Les étales de courant et de niveau coïncident.

Les courants de marée peuvent être alternatifs ou giratoires. Dans le premier cas, ils conservent une même direction pendant le flot et une direction opposée pendant le jusant. Dans le second cas, pendant une période de marée, ils peuvent prendre successivement toutes les directions.

b) Les courants dus aux marées internes ( « internal waves» des Anglo-Saxons). IIs sont en général faibles. Le processus de formation de tels courants est trop peu connu pour que l'on puisse en parler en toute connaissance de cause.

\section{LES CONSEQUENCES DES COURANTS MARINS}

Les courants marins ont des influences diverses et souvent importantes se manifestant dans différents domaines.

La connaissance des courants est de première importance en navigation, aussi les cartes hydrographiques françaises portent la direction et la vitesse des courants. Ces courants ont pour con- 
séquence de faire dévier de leur cap les bâtiments se trouvant sur leur trajectoire. Les grands courants océaniques, tels que le Gulf Stream, sont bien connus des navigateurs. Ainsi la traversée de l'Atlantique est plus rapide d'ouest en "est que d'est en ouest.

Les courants froids, provenant dés mers polaires, transportent des icebergs, ces immenses montagnes de slace qui sont un constant danger pour la navigation. Dans I'hémisphère boréal, une flottille spéciale, la lce Patrol a pour mission de repérer et de signaler la position et la trajectoire des icebergs. Le danger que présentent ces blocs de glace est actuellement amoindri grâce au radar qui permet, aux bâtiments, de les repérer la nuit ou par temps de brouillard.

Les grands courants océaniques, transportant d'énormes masses d'eau, ont pour effet de réchauffer ou de refroidir les côtes qu'ils baignent. Les études sur l'équilibre thermique de notre planète ont permis de déterminer les quantités de chaleur reçues et perdues le long d'un méridien. Ainsi, à l'Equateur, un centimètre carré reçoit 0,339 calorie-gramme par minute et n'en perd que 0,300 , tandis qu'au pôle, il reçoit 0,140 et en perd 0,220 . Pourtant la moyenne annuelle de la température, en différentes latitudes, reste constante ce qui prouve que l'atmosphère et surtout les océans rétablissent l'équilibre. Dans les mers, ce sont les courants qui transportent la chaleur de l'Equateur vers les pôles. Si la Terre était immobile, ces courants suivraient les méridiens mais, la rotation terrestre les dévie vers la droite dans l'hémisphère nord et vers la gauche dans l'hémisphère sud. Ainsi le Gulf Stream, amenant les eaux chaudes des régions équatoriales, réchauffe les côtes de l'Europe occidentale, de la Norvège et permet au Spitzberg d'être dégagé de glaces pendant la plus grande partie de l'année. L'afflux d'eau dans les régions polaires est compensé par un courant froid qui coule le long de la côte du Groënland, refroidissant le littoral de la partie septentrionale des Etats-Unis. C'est le courant du Labrador, grand transporteur d'icebergs.

Si le Gulf Stream pouvait être dévié de sa trajectoire actuelle, il ne réchaufferait plus les côtes norvégiennes, celles-ci deviendraient semblables au rivage groënlandais, une calotte de glace recouvrirait les montagnes scandinaves et le port de Brest pourrait même être bloqué par les glaces pendant une partie de l'année, comme à la même latitude sur la côte américaine. On voit, d'après cet exemple, l'influence climatique considérable des courants marins.

Les courants littoraux, quelle qu'en soit l'origine, présentent un intérêt primordial pour l'hydrographe, le géologue et l'ingénieur des Travaux Publics. En effet ces courants transportent des matériaux solides, sable ou vase, qu'ils arrachent à un point de la côte, où elle s'érode, pour les déposer en un autre point où elle s'engraisse. Plus exactement, il vaudrait mieux dire que la houle met en suspension les matériaux et qu'ils sont ensuite transportés par les courants (en employant ce terme dans le sens d'écoulement d'ensemblel. L'envasement et l'ensablement des ports, s'ils sont quelquefois le résultat d'une houle persistante, sont le plus souvent dû à des courants de marée. Qu'il nous suffise de citer le cas du port de Honfleur présentant, en 1945, à marée basse, l'aspect d'une série de monticules de vase ravinés par les eaux de ruissellement. L'étude des courants littoraux est donc d'un grand intérêt et doit être effectuée avant toute construction douvrage de protection. Les courants doivent également être mesurés après l'édification de ces ouvrages pour déterminer leur réaction sur le régime général des courants.

Les spécialistes des pêches ont remarqué qu'à certairies époques les poissons migrateurs se tiennent généralement en des points bien définis. Souvent ces régions sont celles où les eaux chaudes se mélangent aux eaux froides des rands courants. L'étude des courants et la détermination des points de mélange des eaux peuvent donc être d'une certaine importance pour les pêcheurs.

\section{DETERMINATION DES COURANTS MARINS}

Ces déterminations peuvent être indirectes ou directes. Les premières sont des calculs à partir des caractéristiques (température et salinité) de l'eau de mer en des points différents, tandis que les secondes sont des mesures et impliquent l'usage d'un appareillage spécial. II faut remarquer que les déterminations indirectes ne peuvent s'appliquer qu'aux courants de densité, tandis que les méthodes directes sont utilisables pour les courants de toute nature.

\section{Détermination indirecte des courants marins.}

A un moment donné et en un point bien déterminé de l'Océan, l'eau de mer se trouve définie par sa température et sa salinité. A partir de ces deux valeurs, et en tenant compte de la profondeur, les tables donnent la densité " in situ».

En joignant les points de même pression, on obtient des courbes isobariques. L'ensemble de ces courbes constitue des surfaces isobariques.

Ce sont des surfaces imaginaires le long desquelles la pression, dans le fluide, reste constante. Les surfaces de niveau sont des surfaces le long desquelles ne s'exerce aucune composante de l'accélération de la pesanteur. Si les surfaces isobariques et les surfaces de niveau coïncident, le gradient de pression, dirigé de bas en haut, équilibre l'accélération de la pesanteur par unité 
de masse ; l'équilibre statique est alors parfait et le fluide est absolument immobile. Cette coincidence est rarement réalisée; les deux surfaces forment entre elles un angle faible, une composante de la pesanteur agit le long de la surface isobarique et le fluide ne peut plus rester au repos, il se meut en descendant la surface en pente mais aussitôt intervient la force de déviation due à la rotation de la Terre (force de Coriolis). Elle est dirigée vers la droite dans l'hémisphère Nord et vers la gauche dans l'hémisphère Sud. Ainsi dans l'hémisphère boréal les eaux les plus légères se trouvent sur la droite d'un obervateur regardant dans le sens du courant, tandis que les eaux les plus lourdes se placent à gauche.

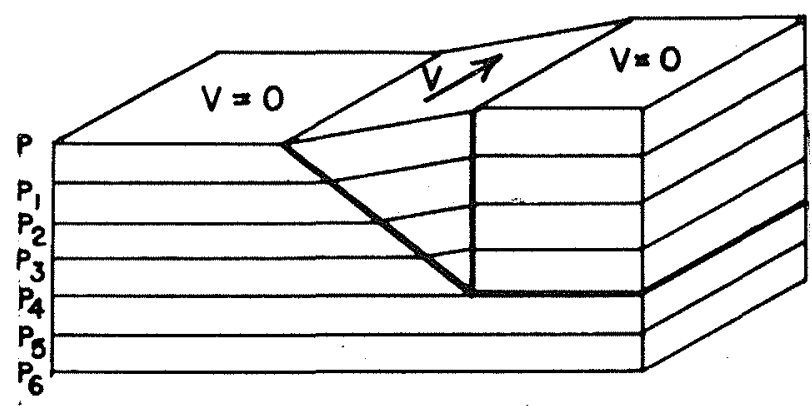

Fig. 3

Représentation schématique d'une série de surfaces isobariques.

Pour déterminer la vitesse absolue des courants ainsi créés on admet qu'on peut prendre une surface isobarique de référence le long de laquelle les courants sont nuls, c'est-à-dire où une surface isobarique coincide avec une surface de niveau. L'unité de mesure de pression, employée en océanographie, est le décibar, correspondant sensiblement à la pression exercée par une colonne d'eau de 1 mètre de haut. Ayant ainsi choisi une surface de référence à 2.000 mètres de profondeur, on dira qu'on se réfère à la surface de 2.000 décibars.

La détermination des courants, par cette méthode, est sujette à un certain nombre d'erreurs En premier lieu, on peut avoir mal choisi la surface de référence; en second lieu, les mesures de salinité et de température, qui sont à la base de la construction des surfaces isobariques, ne sont pas simultanées d'un point à un autre de l'Océan, pourtant, dans le calcul, on est obligé de les considérer comme simultanées.

II s'introduit également une certaine erreur, d'ailleurs faible, en négligeant les forces de frottement et en supposant que les pentes des surfaces dépendent exclusivement de la densité et qu'en général on peut trouver une surface isobarique de niveau à laquelle toutes les pentes sont référées.

Malgré tous ces défauts, la méthode dynamique de détermination des courants est très satis. faisante. Ses résultats, comparés à ceux obtenus par la méthode directe, ont donné un bon accord. Dans le détroit de Floride, il a été effectué des déterminations par le calcul et des mesures directes. Les écarts ont été assez faibles.

Pour que la valeur de la vitesse et de la direction du courant calculé ait une signification et puisse être utilisée par tous les océanographes, il faut essentiellement que les mesures de la température aient été faites ovec une précision de $0,01^{\circ} \mathrm{C}$. et les dosages de salinité à $0,01 \%$ près. Il faut également que les observations aient été effectuées au cours d'une même campagne et ne soient séparées que par un intervalle de temps aussi court que possible. Si la zone est telle que les courants ont une permanence et une régularité marquées, on peut faire des mesures espacées dans le temps.

\section{Détermination directe des courants marins.}

Cette opération exige :

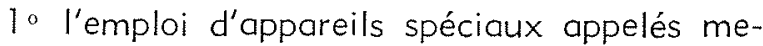
sureurs de courant (courantomètre).

$2^{\circ}$ un point fixe auquel sont fixés les appareils. Ce point fixe peut être une bouée, un corps ou un bâtiment ancré. Dans ce cas on effectue des mesures au point fixe. Si on utilise des flotteurs dérivants, on matérialise ainsi les filets fluides.

D'après leur mode d'utilisation, on peut classer les mesureurs de courant à point fixe en deux catégories suivant qu'ils sont immergés d'un bâtiment ou abandonnés en mer après avoir été fixés à une bouée ou à un corps mort. Ce sont les appareils de la première catégorie qui sont plus communs et les plus employés, tandis que ceux de la deuxième sont plus rares. Pourtant, parmi ces derniers, il existe un bon appareil idû à l'ingéniosité de P. IDRAC et fabriqué en France.

D'après leur mode d'enregistrement, on peut également classer les appareils existants en deux groupes, suivant qu'ils ont un enregistrement faisant partie de l'appareil ou bien un système d'enregistrement à distance placé à bord du bâtiment qui supporte le courantomètre. Les appareils abandonnés en mer ont un enregistrement faisant partie intégrante de l'instrument à l'exception du mesureur de courant de ROBERTS dont l'enregistreur est porté par la bouée.

Nous avons indiqué que l'emploi des mesureurs de courant exige un point fixe absolu. Pour les appareils abandonnés en mer, qu'ils soient fixés à une bouée ou à un corps mort, le problème ne souffre aucune difficulté, mais malheureusement de tels appareils ne peuvent travailler par grands fonds, car ils exigeraient un câble très long dont le poids serait considérable. Pour les appareils suspendus au bâtiment et qui exigent 
l'emploi de messagers destinés à mettre en route, puis à bloquer les mécanismes d'enregistrement, i) est indispensable d'assurer l'immobilité du navire. Par petits fonds, en général inférieurs à 200 m., le problème est relativement soluble moyennant quelques précautions, mais pour les grands fonds, il se complique sérieusement. Le seul moyen pour rendre immobile un bâtiment est de l'ancrer. Par des fonds de plusieurs milliers ide mètres, cette manœuvre exige un treuil puissant, une grande longueur d'un câble solide et une ancre ( ou un corps mort) d'un poids important. L'immobilité d'un navire, ancré par grands fonds, est pratiquement illusoire, car si le courant est violent ou si le vent souffle l'ancre dérape et le bâtiment se meut dans le sens du courant ou du vent. La solution de mouiller deux ancres est meilleure, mais ne donne pas une sécurité absolue. Même en supposant que l'ancre, ou les ancres, soient immobiles sur le fond, le bâtiment lui-même, à la surface de l'Océan, n'est pas immobile. Il subit trois mouvements : le rappel qui le rapproche et l'éloigne du point de contact de l'ancre avec le fond, l'évitage qui lui fait décrire un arc de cercle autour de l'ancre (mouvement en partie supprimé si on mouille deux ancres) et les embardées qui lui font faire des mouvements désordonnés autour du point de contact du bâtiment et de la chaîne de l'ancre.

Si le bâtiment est mouillé hors de vue de terre, il lui est impossible de vérifier sa position avec exactitude et très souvent il peut dériver sans que l'observateur s'en rende compte. Les résultats des mesures directes de courant, qui ont été publiés, sont souvent sujets à caution car il est impossible d'être assuré que le bâtiment était absolument immobile.

Pourtant certains océanographes ont pu mouil ler par grands fonds et affirment avoir obtenu l'immobilité de leurs navires. En 1888, Pillsburg mouilla son bâtiment, le Blake, par 3.000 mètres de profondeur. En 1926, le navire allemand Météor effectua, avec succès, la même opération par 5.500 mètres. Juste avant la guerre, le bâtiment américain A tlantis resta à la même place plus de cinq jours par des profondeurs supérieures à 5.000 mètres. Mais si l'opération du mouillage a pu être menée à bien à bord de ces bâtiments, rien ne prouve que ceux-ci étaient absolument immobiles au cours des observations océanographiques.

Les Norvégiens se proposent de mettre au point un procédé nouveau de mesure directe des courants marins. Le mesureur de courant une fois immergé, ils détermineront avec précision la position du navire à l'aide de stations de DECCA, en même temps qu'ils déclencheront le mécanisme de fonctionnement de l'enregistreur de l'appareil. IIs laisseront dériver leur bâtiment, sous l'effet des courants superficiels ou du vent, et, au bout d'un temps assez long, ils détermineront de nouveau leur position en même temps qu'ils arrêteront l'enregistreur du courantomètre. Connaissant la direction et la vitesse absolues fournies par le mesureur de courant et la composante due à la dérive, ils pourront mesurer la composante due au courant qui se fait sentir à la profondeur à laquelle a été immergé l'appareil. Cette méthode ne peut donner des résultats acceptables que si les deux positions sont connues avec une grande précision ce qui peut être obtenu avec un réseau de stations de DECCA. De toutes manières, on ne peut avoir, par ce procédé, qu'une valeur moyenne de la direction et de la vitesse du courant le long de la trajectoire parcourue par le bâtiment au cours de sa dérive.

\section{DESCRIPTION \\ DES PRINCIPAUX APPAREILS EXISTANTS}

D'après le principe sur lequel est basé leur fonctionnement, les appareils existants peuvent être divisés en six catégories : les moulinets, les pendules, les tubes de Pitot, les appareils utilisant la résistance à l'avancement, les fils chauds et les flotteurs.

\section{Les moulinets.}

Parmi ces appareils, certains possèdent des hélices à axe parallèle à celui du courant, d'autres sont pourvus de coupelles portées par un axe perpendiculaire à la direction du courant. L'enregistrement de ces instruments est soit mécanique, soit électrique, soit photographique. Dans certains mesureurs de courant, l'ingéniosité des inventeurs a associé deux de ces procédés.

Tous les courantomètres, quel que soit leur mode d'enregistrement, doivent être étalonnés dans des conditions identiques à celles de leur utilisation normale.

L'appareil le plus connu et le plus couramment utilisé dans le monde est celui conçu par EKMAN (1932) (fig. 4). Il est essentiellement constitué d'une hélice solidaire d'un compteur de tours, d'une boussole et d'une dérive. L'enregistrement est entièrement mécanique sans boîte étanche.

L'hélice, à huit pales, tourne à l'intérieur d'un anneau de garde. Sa rotation est transmise à un compteur à deux cadrans, placé sur le côté gauche de l'appareil. La boussole constitue une particularité ingénieuse de cet instrument. Chaque fois que l'hélice a effectué 100 rotations une bille provenant d'un magasin tombe au centre de l'aiguille aimantée, court le long d'une rigole et tombe dans l'un des 36 compartiments qui divisent la boussole. La dérive est constituée par une plaque métallique verticale reliée à l'appareil par deux tiges horizontales. Le mesureur de courant est suspendu à un filin, lesté à sa partie inférieure, et descendu à la profondeur désirée. On 
envoie alors un messager qui ouvre les volets $F$, protecteurs de l'hélice et débloque celle-ci. Au bout d'un temps déterminé, on envoie un second messager qui referme les volets et bloque l'hélice. L'appareil ramené à bord, il suffit de lire le compte-tours pour connaître la vitesse et de compter les billes des divers compartiments du compas pour apprécier la direction.

L'appareil d'EKMAN est simple, robuste, stable, sensible et fidèle. 11 permet de mesurer les vitesses comprises entre 5 et $250 \mathrm{~cm}$./sec. L'hé-

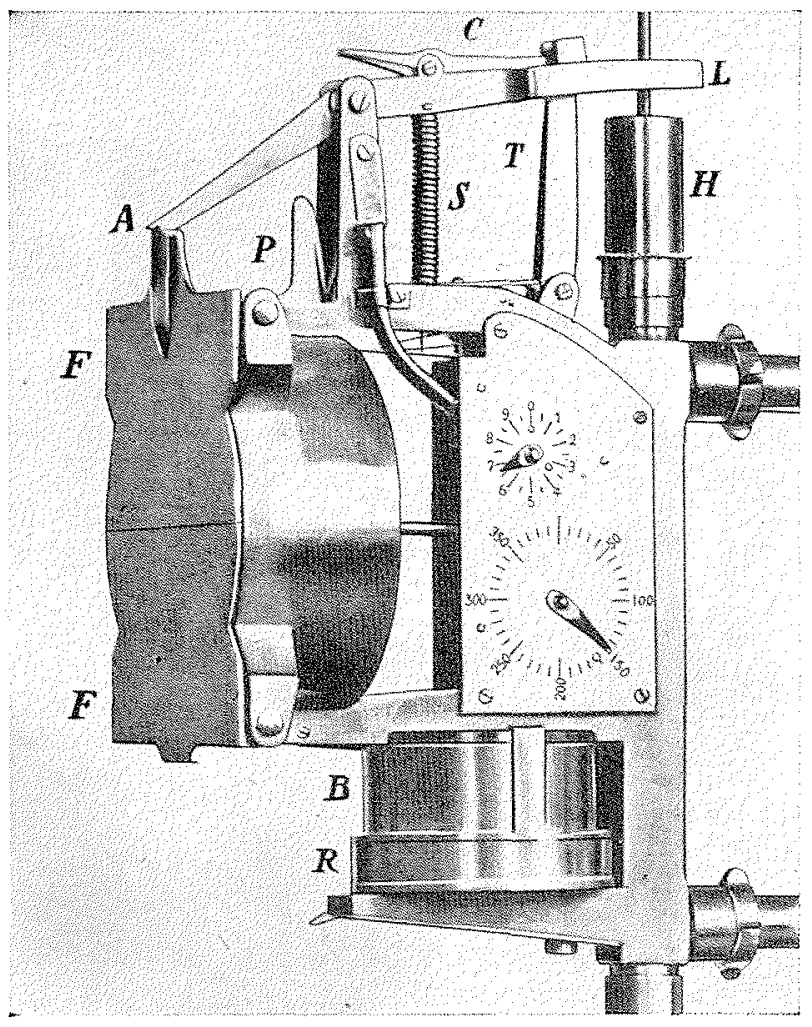

Appareil d'Ekman. Volets fermés.

lice de l'appareil étant très légère $(40 \mathrm{gr}$.), il n'y a qu'un faible effet de volant et son inertie est faible. Pourtant il présente quelques inconvénients :

10 il ne peut être abandonné en mer, car il exige un messager qui implique une présence humaine au début et à la fin de la mesure:

$2 \circ$ les volets protecteurs ouverts en amont de I'hélice apportent une perturbation dans l'écou. lement surtout si la direction du courant s'écarte même légèrement de l'axe de l'hélice:

$3^{\circ}$ l'enregistrement mécanique, étant entièrement au contact de l'eau de mer, risque des corrosions qui modifient les caractéristiques de l'appareil fixées par étalonnage.

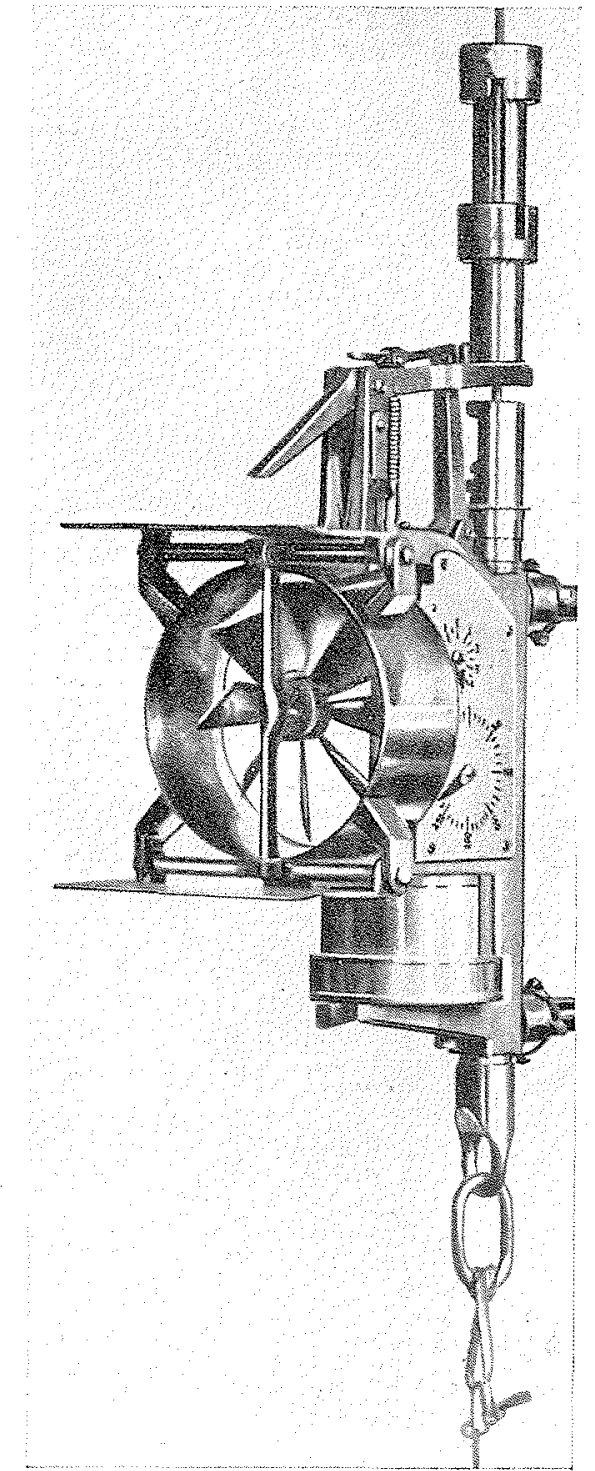

Fig. 4

Appareil d'Ekman. Volets ouverts.

L'appareil de BOHNECKE fut utilisé par les Allemands en 1937, à bord du Meteor. De tracé pisciforme, il porte, à l'avant, une hélice entourée d'un anneau de protection et, à l'arrière, quatre plans de dérive. Les organes indicateurs de la vitesse de rotation de l'hélice et de la direction, par rapport à un compas, sont constitués par des disques horizontaux munis de chiffres à leur périphérie. Un mouvement d'horlogerie fait passer une mince bande de zinc devant ces disques. Un coup de marteau, produit à intervalles réguliers, appuie cette bande contre les disques et imprime les chiffres correspondants à la vitesse et à la direction au moment de la frappe. Tout le mécanisme d'enregistrement est baigné par l'eau de 
mer à l'exception du mouvement d'horlogerie. Cet appareil ne peut fonctionner que pendant douze heures avec un enregistrement toutes les cing minutes, mais il ne semble pas difficile, avec des modifications appropriées, de prolonger le temps d'enregistrement. II peut ainsi être abandonné en mer après avoir été fixé à une bouée. Mais nous n'avons que peu de renseignements sur son emploi, aussi il est difficile d'en faire la critique en toute connaissance de cause.

Entre 1918 et 1925, H.-U. SVERDRUP utilisa, au cours de la campogne du Malld dans les régions polaires, un mesureur de courant à enregistrement électrique. Pour celui des vitesses, une hélice, à axe horizontal, fait tourner, par l'intermédiaire d'une vis sans fin, une roue qui ouvre et ferme un circuit électrique. La direction du courant est donnée par rapport à un support qui reste parallèle à l'axe du bâtiment. Un bras, relié à un des pôles d'une source d'énergie et solidaire du mesureur de courant, balaie une résistance électrique reliée à l'autre pôle. Ainsi l'intensité du courant donne la direction. Les organes électriques vitaux sont protégés par un bain de pétrole placé à la partie supérieure de l'appareil.

C'est la première tentative en vue de l'utilisation d'un circuit électrique dans l'enregistrement des vitesses et des directions. Malheureusement cet appareil ne permet pas de mesurer des vitesses inférieures à $10 \mathrm{~cm}$./ $\mathrm{sec}$.

L'appareil de OTT (1933) est également à enregistrement électrique, mais celui-ci n'est pas continu et s'obtient par le jeu d'un contact placé à portée de l'observateur. Les organes délicats de l'appareil sont plongés dans un bain de pétrole. Deux conducteurs sont nécessaires aux divers circuits électriques. Ces derniers sont d'un usage peu pratique en océanographie, car chaque immersion et chaque remontée de l'appareil obligent à les dérouler et à les enrouler sur des tambours, ce qui présente l'inconvénient de détériorer les isolants.

Le courantmètre de RAUSCHELBACH (1929) rappelle celui de OTT, mais il est plus compliqué et plus délicat.

Tout dernièrement (1947), I'U. S. Coast Guard publia la description d'un mesureur de courant dû à ROBERTS (fig. 5). Il est destiné à mesurer les caractéristiques du courant en des points où il peut être abandonné, simplement suspendu à une bouée de forme spéciale. Sa principale particularité réside dans la transmission des résultats d'enregistrement par radio. L'appareil lui-même, de forme fuselée, porte, à l'avant, une hélice à axe horizontal. Sa rotation est transmise, par un couple magnétique, à un mécanisme d'enregistrement placé dans un compartiment étanche à l'abri des corrosions de l'eau de mer. Le mécanis- me opère des contacts qui sont transmis, par câbles électriques, jusqu'à la bouée, celle-ci étant pourvue d'un poste émetteur ef d'une antenne, diffuse les résultats qui sont reçus, à plusieurs miles de distance sur un chronographe central. Il est possible d'avoir plusieurs courantomètres disséminés dans la région à étudier, car chaque appareil émet sur une longueur d'onde séparée. Les utilisateurs, après les premiers essais, se proposent d'apporter des améliorations à cet ensemble ingénieux dont le système d'enregistrement et de transmission présente de réels avantages sur ses prédécesseurs. Malheureusement, actuellement, un tel ensemble comprenant le courantomètre, la bouée et le poste émetteur coûte plus de deux millions de francs.

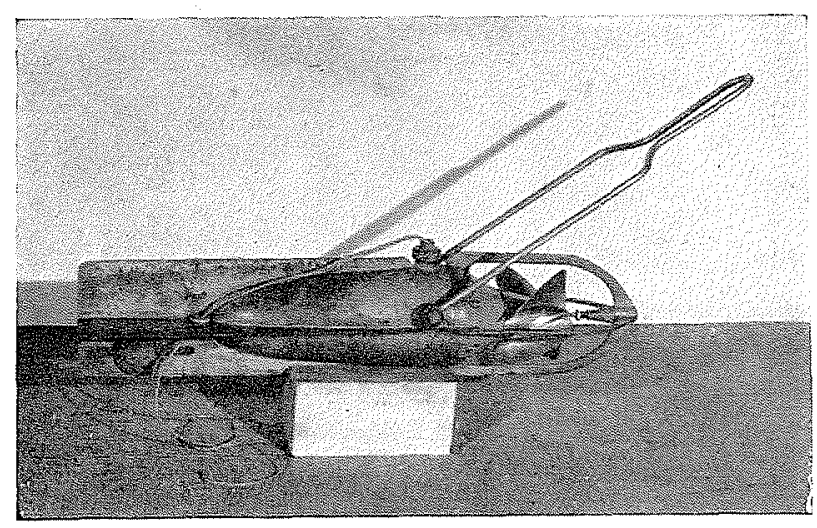

Fig. 5

Cliché Marine Instrument.

Appareil de Roberts.

Le premier appareil, de conception très simple, utilisant un enregistrement photographique de la direction est dû à O. PETTERSON (1913). La direction est indiquée par une aiguille à compas portant un tube fin rempli d'une substance radioactive. L'image de cette source de "lumière», qui tourne avec la direction du courant, apparaît sur une plaque photographique enfermée entre deux feuilles de celluloïde collées ensemble. L'enregistrement de la vitesse n'apporte aucune novation. Le seul intérêt de cet appareil réside dans son curieux mode d'enregistrement de la direction.

Le Musée Océanographique de Monaco possède un mesureur de courant dû à l'Italien BOCCARDO (fig. 6). II est d'une construction très soignée et d'une conception assez séduisante. Quatre coupelles à axe horizontal (mais perpendiculaire à la direction du courant) tournent dans un plan vertical. L'axe est supporté par des pivots en pierre dure. La rotation est transmise à un compte-tours, situé sur le côté droit de l'appareil. Le 
bâti porte, à sa partie supérieure, un compos qui se bloque à la fin de la mesure et donne ainsi la

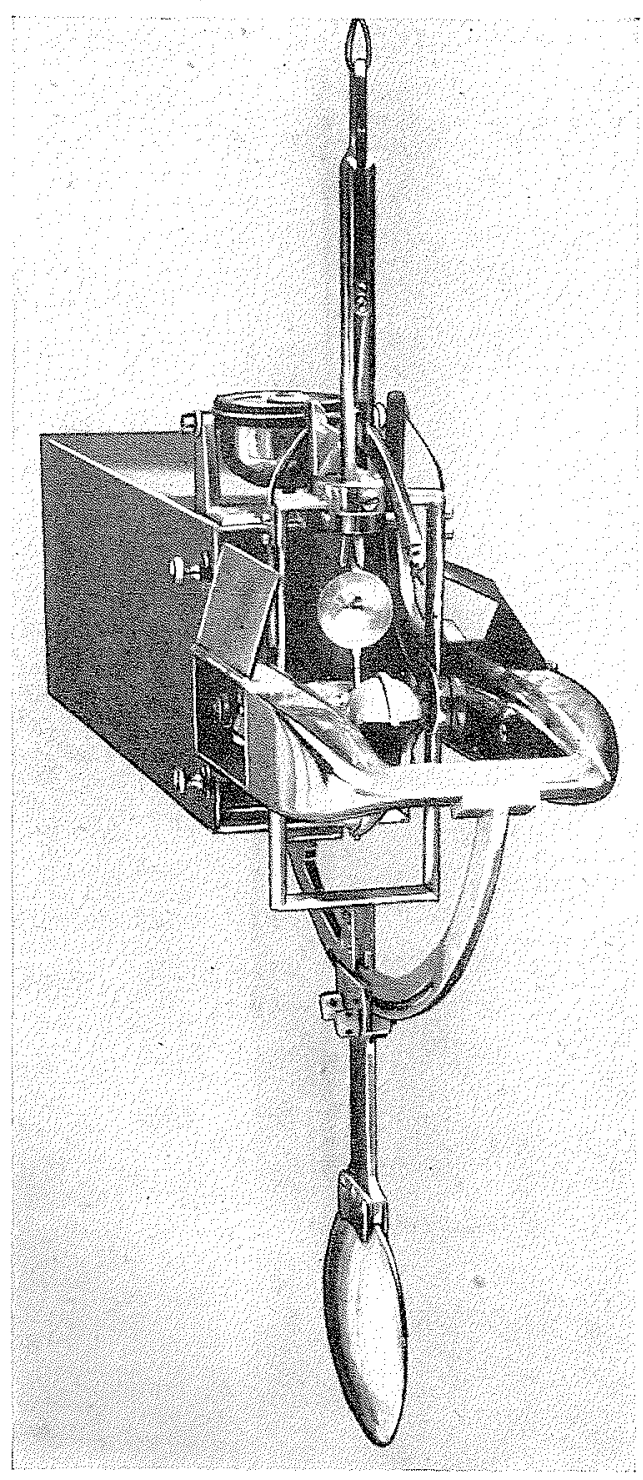

Apporail de Boccardo. lisation de cet appareil n'a été publié, aussi il est difficile de savoir quel peut être son comportement réel. A première vue, il semble être d'une construction très soignée, mais il présente tous les inconvénients des appareils à coupelles. II ne peut donner qu'une valeur moyenne de la vitesse et la direction n'est connue qu'à l'instant où le second messager bloque le compas, ce qui constitue un grave défaut de l'appareil.

En France, on utilise le courantomètre dû à $P$. IDRAC (1931). C'est un appareil à moulinet dont l'axe est vertical. Son enregistrement, faisant partie de l'instrument, utilise un film cinématographique pour la détermination de la vitesse et de la direction.

Le mesureur de courant de P. IDRAC est essentiellement constitué (fig. 7) d'un châssis, servant de carter à un moulinet $A$, qui supporte deux $c y$ -

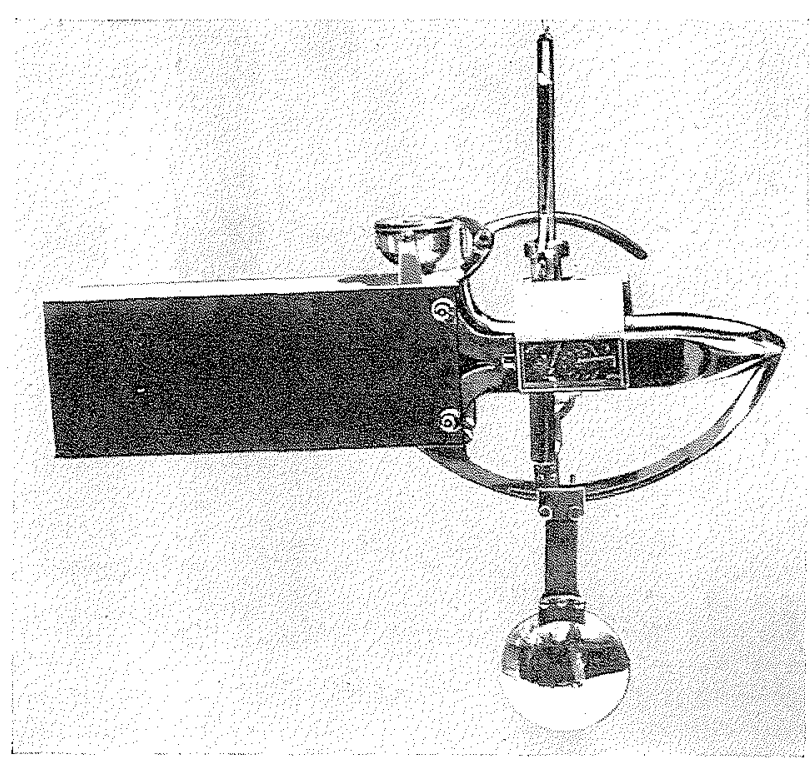

Fig. 6 direction. La dérive a une forme de tunnel où l'eau s'écoule après avoir passé sur le moulinet. L'appareil possède, à sa partie inférieure, un poids de lestage en forme de lentille. Le moulinet, très léger, est en aluminium, tandis que toutes les autres pièces sont en bronze nickelé. Un messager, envoyé au début de la mesure, débloque le moulinet et le compas. Un autre messager, à la fin de la mesure, bloque le moulinet et l'aiguille aimantée de la boussole en renversant celle-ci. A notre connaissance, aucun compte rendu d'uti- lindres extérieurs étanches B et un cylindre central C. L'un des cylindres extérieurs renferme la batterie d'accumulateurs $J$, tandis que l'autre contient un compas $L$, un carter cloisonné avec trois lompes $M$ et un appareil photographique amovible N. Celui-ci, grâce à un mouvement d'horlogerie, fait défiler un film de $35 \mathrm{~m} / \mathrm{m}$. devant un objectif $O$. Le cylindre central $C$ est ouvert vers le bas. Dans sa partie supérieure, il porte des contacts électriques, en liaison avec le moulinet à six bras portant chacun une coupelle 
hémisphérique. Un bain d'huile protège les systèmes démultiplicateurs $Q$ et les contacts électriques. Pour permettre à l'appareil de s'orienter dans le courant, il est pourvu d'une queue en bois.

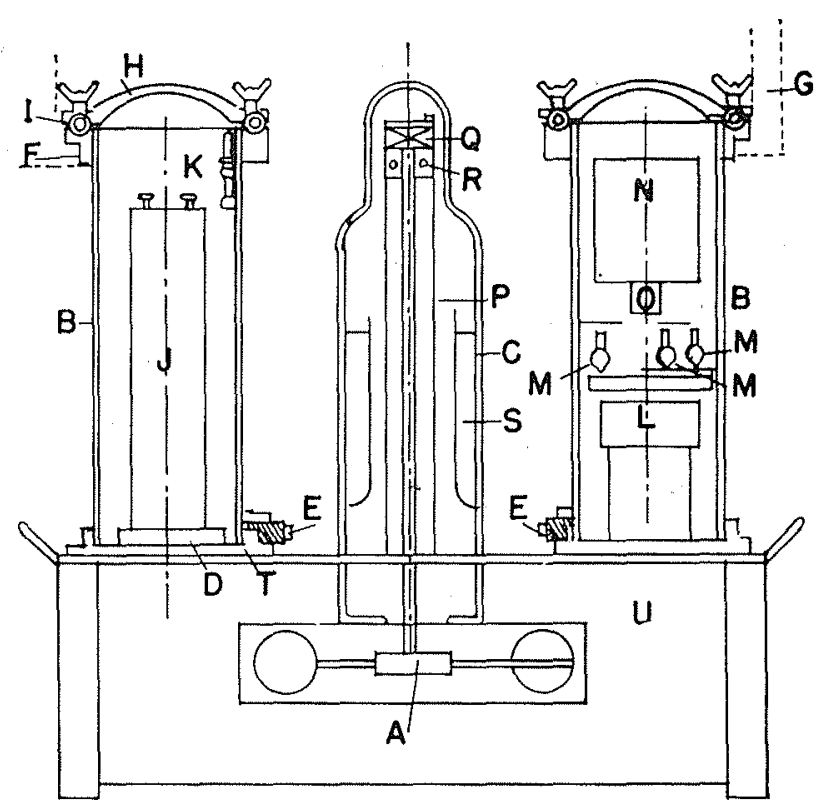

(A)

Coupe schématique de l'appareil de P. Idrac.
Cet ensemble de traits est projeté, à l'aide d'un objectif, sur une fente placée devant le film. Le cadran de la boussole restant orienté nord-sud, quelle que soit l'orientation de l'appareil, le film

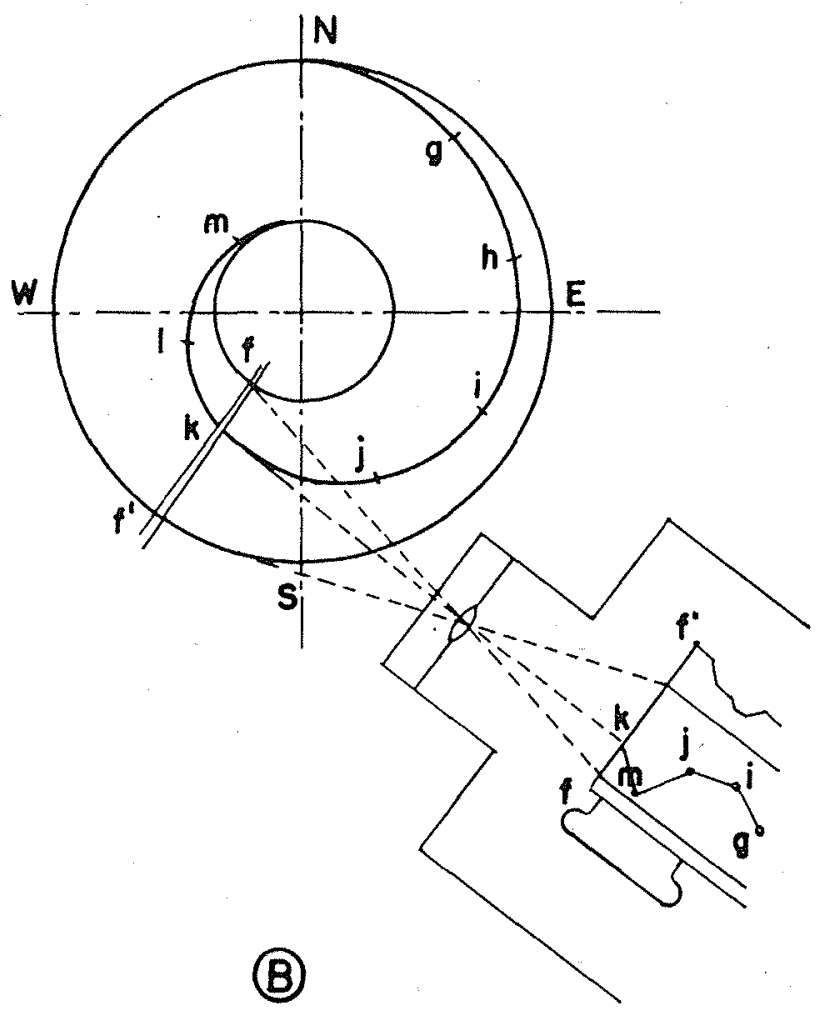

Enregistrement de la direction

Fig. 7

L'enregistrement des vitesses est obtenu par la rotation du moulinet. Son arbre, par un système d'engrenages démultiplicateurs, actionne un doigt qui ferme, pendant quelques secondes, tous les n tours, un circuit électrique. Celui-ci provoque l'allumage d'une petite lampe électrique qui impressionne le film se déroulant, à vitesse constante, derrière l'objectif. Pour obtenir plus de précision de lecture, les inscriptions de la vitesse sont faites à deux échelles, le moulinet allumant une lampe tous les $n$ tours et une autre tous les $5 \mathrm{n}$ tours. On obtient ainsi deux séries de traits, les uns étroits et rapprochés, les autres plus larges et plus espacés. La direction est enregistrée sur la même pellicule. Pour cet enregistrement, il est utilisé un compas portant trois traits en blanc sur fond noir; deux d'entre eux sont des cercles concentriques tracés autour du pivot, tandis que le troisième est un trait en spirale partant du nord pour revenir au nord comme l'indique la figure $7(B)$. enregistrera trois traits. Les traits extrêmes, re. présentant les cercles concentriques, seront parallèles entre eux, tandis que le trait intermédiaire sera plus ou moins éloigné des deux' autres suivant la portion de la spirale qui se trouvera dans le champ de la fente.

Nous avons décrit en détail l'appareil de $P$. IDRAC car c'est un bon courantomètre fabriqué en France. II présente néanmoins quelques défauts qu'il n'est pas inutile de souligner. II ne donne que des vitesses moyennes et ne permet pas la mesure des faibles courants. Le moulinet se trouve enfermé dans un tunnel; ainsi, ou lieu de travailler en veine illimitée, il tourne en veine limitée, ce qui modifie les conditions d'écoulement si le nombre de Reynolds vient à changer. Son enregistrement des directions est très acceptable et, en apportant quelques améliorations à son mode d'enregistrement des vitesses, on peut avoir là un excellent appareil. 


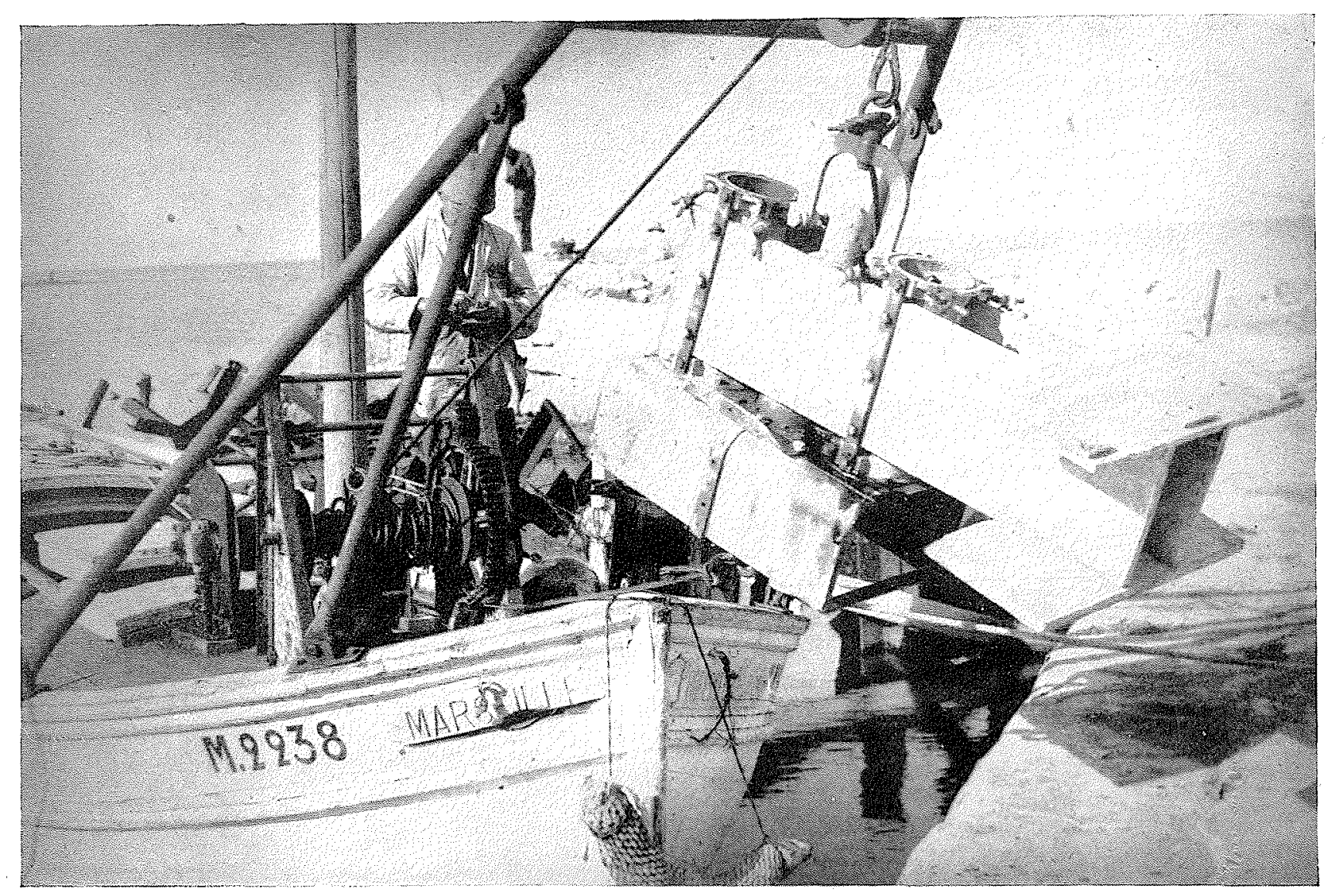

Fig. 8. - Appareil de P. Idrac.

\section{Les pendules.}

A notre connaissance, il n'existe que deux appareils basés sur les oscillations d'un pendule soumis, directement ou indirectement, à l'action d'un courant. Ces instruments ne sont plus actuellement utilisés, mais ils comportent, dans leur principe, une idée intéressante (fig. 9 A et 9 B).

En 1906, F. NANSEN publia la description d'un mesureur de courant utilisant les oscillations d'un pendule soumis directement à l'action du courant marin. II est essentiellement constitué par un pendule à ailettes suspendu à un levier. Un mouvement d'horlogerie fait tourner une roue dentée qui abaisse, puis relève le pendule. Lorsqu'il est levé, il peut osciller librement et prendre une position d'équilibre sous l'influence du courant, mais quand la roue dentée l'abaisse, sa pointe touche la surface, recouverte de cire, d'un disque concave et y laisse une trace ponctuelle. Le disque est solidaire d'une aiguille aimantée qui tourne sur un pivot. II porte une flèche qui coïncide avec le nord magnétique, aussi est-il toujours parfaitement orienté. F. NANSEN disposait de trois pendules de forme et de poids divers.
L'un de ces pendules lui permit de mesurer des vitesses de $0,5 \mathrm{~cm}$. $/ \mathrm{sec}$.

Cet appareil présente de notables défauts. N'étant pas parfaitement stable dans le courant, ses déplacements apportent une cause d'erreurs difficiles à chiffrer. Les montants verticaux du mesureur de courant, reliant le mécanisme de suspension du pendule au disque, créent des perturbations qui influent sur les oscillations du pendule. Toutes les pièces métalliques étant en contact avec l'eau, sont appelées à être oxydées, particulièrement le mouvement d'horlogerie et le pivot de la boussole. Par ailleurs, tout corps dérivant dans l'eau et entrant en contact avec le pendule a pour effet de fausser totalement les mesures. Le seul avantage de cet appareil est de pouvoir mesurer les très faibles vitesses, mais malheureusement il ne peut en donner que la valeur moyenne et les extrêmes.

Le bathyrhéomètre de $Y$. DELAGE (1912) utilise également les oscillations d'un pendule, mais, dans cet appareil, le pendule lui-même est enfermé dans une sphère étanche qui s'incline sous l'effet du courant. Contrairement au courantomètre de F. NANSEN, celui de Y. DELAGE pré- 


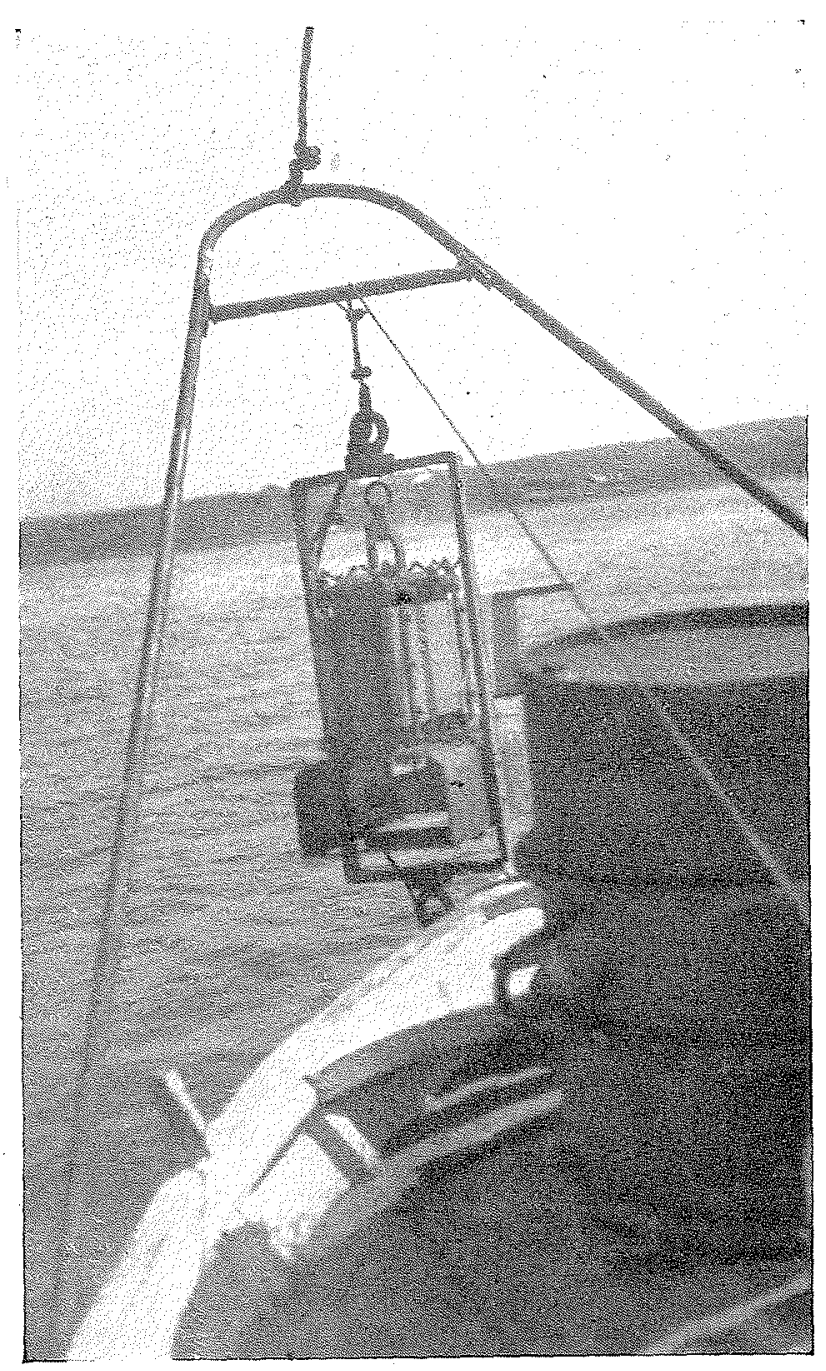

Fig. 8 bis

Appareil de P. Idrac suspendu à une chèvre à I'avant du bâtiment et prêt à être immergé.

sente un ingénieux dispositif d'enregistrement mécanique utilisant I'association de deux mouvements d'horlogerie engendrant des mouvements situés dans les deux plans perpendiculaires.

A première vue, cet appareil, malgré ses quelques défauts, est très séduisant. Malheureusement il a été très peu étudié. II semble que des études pourraient être poursuivies en vue d'arméliorer et de mettre au point un nouveau bathyrhéomètre perfectionné. De toutes manières cet instrument ne pourra donner les caractéristiques du courant que près du fond car il est impossible d'allonger indéfiniment la tige, ou le câble, qui le relie au fond sous-marin.

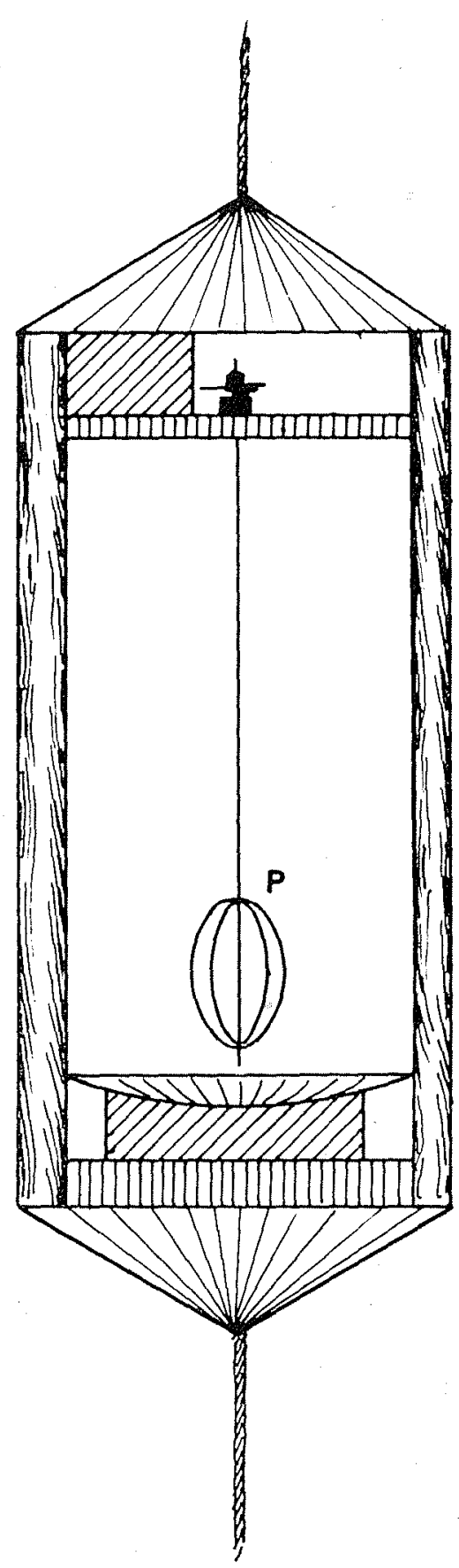

(A)

Fig. 9

Schéma des pendules utilisés en océanographie.

A. - Appareil de F. Nansen dans lequel le pendule P s'incline sous l'effet du courant, le bâti restant immobile. 


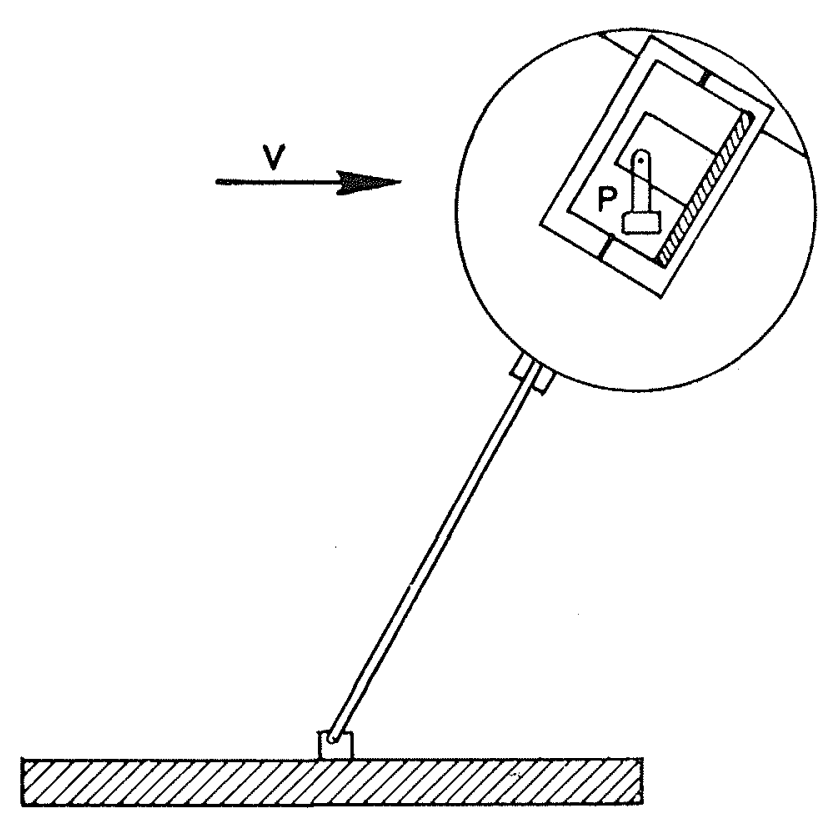

(B)

Fig. 9

Schéma des pendules utilisés en océanographie.

B. - Appareil de $Y$. Delage dans lequel le pendule $P$ reste toujours vertical, la sphère s'incline sous l'effet du courant $V$.

\section{Les tubes de Pitot.}

Les tubes de Pitot ne sont guère utilisables en océanographie car leur inertie est considérable et leur sensibilité très réduite. Certains auteurs ont essayé d'améliorer les tubes de Pitot pour permettre leur utilisation dans les essais sur modèles réduits. II semble que dans ce domaine, ils peuvent rendre quelques services par suite de la faible perturbation apportée par l'antenne de prise de pression ce qui permet de la placer le long des modèles.

Y. DELAGE, en 1920, essaya de construire un appareil, basé sur le principe du tube de Pitot, destiné à des recherches océanographiques. II remplaça la mesure de hauteur manométrique par une mesure de débit; mais son appareil, malgré toute l'ingéniosité qui a présidé à sa construction, ne peut fonctionner qu'en eau absolument calme; dans ce cas, il donne une valeur moyenne de la vitesse et aucune indication sur la direction. Il ne présente absolument aucun intérêt pour l'océanographe.

\section{Les appareils utilisant la résistance à l'avancement.}

WOLLASTON (1930) construisit un appareil constitué par deux disques perforés qui, grâce à un contre poids et une dérive, restent constamment perpendiculaires à la direction du courant. Ces disques sont solidaires d'un tambour étanche qui peut tourner autour de pivots horizontaux. L'inclinaison du tambour est enregistrée sur une bande à déroulement continu mû par un mouvement d'horlogerie. A des intervalles de vingt minutes, la direction est également enregistrée par comparaison avec un compas magnétique. L'appareil possède deux paires de disques interchangeables, l'une pour les courants relativement faibles, l'autre pour les forts. Cet instrument ne peut mesurer que des vitesses supérieures à 12 $\mathrm{cm}$./sec. Il est peu sensible et sa fidélité est subordonnée à un très bon entretien. Il a l'avantage d'être robuste et relativement peu coûteux.

\section{Les fils chauds.}

Depuis de nombreuses années, on utilise en aérodynamique, principalement pour les essais des maquettes en souffleries, des anémomètres à fil chaud basés sur les variations de résistance d'un fil chauffé. Un mince fil d'un métal inoxydable est soumis au courant d'air qui a pour effet de le refroidir, ce qui fait varier sa résistance. Si ce fil fait partie d'un pont de Wheatstone, cette variation de résistance se traduira par un déséquilibre qui sera mesuré par un galvanomètre.

Il a semblé intéressant d'adapter les appareils à fil chaud aux liquides; mais les chercheurs se heurtèrent à des difficultés considérables et jusqu'ici aucun appareil, utilisable en océanographie, n'a pu être construit. Pourtant si on pouvait surmonter les difficultés, il serait possible d'avoir un mesureur de courant possédant des avantages indéniables sur les appareils existants. Grâce à sa faible inertie, un tel courantomètre permettrait de mesurer les vitesses instantanées et de déceler les faibles courants.

\section{Les flotteurs.}

La mesure des courants marins par la méthode des flotteurs est très simple, mais elle conduit à un certain nombre d'inconvénients. C'est incontestablement la plus ancienne méthode que les océanographes aient employée pour se faire une idée des courants océaniques.

L'appareillage est très simple et consiste en un flotteur qui peut être une bouteille lestée, une bouée munie d'un voyant ou tout autre objet flottant. Si on veut mesurer les vitesses et direc- 
tion d'un courant à une certaine profondeur (en général inférieure à vingt mètres), on construit un croisillon en bois ou en métal, relié, par un câble, à un petit flotteur de surface. II est nécessaire que la surface du flotteur de surface soit négligeable par rapport à celle du croisillon pour que l'ensemble soit uniquement influencé par le courant qui se fait sentir à la profondeur à laquelle est immergé le croisillon.

La mesure de la vitesse et de la direction d'un courant par la méthode des flotteurs peut se faire soit en suivant à l'aide de théodolites les flotteurs dans leur course, soit en les laissant dériver sur de très longs parcours et en déterminant leurs positions de départ et d'arrivée. Dans ce dernier cas, les flotteurs matérialisent la trajectoire des filets fluides.

Si la méthode des flotteurs est simple, elle est loin d'être précise. Elle ne donne qu'une vitesse et une direction très moyennes du courant. Le vent, surtout s'il est violent, a une grosse influence sur le flotteur de surface et sur son voyant. Par ailleurs, cette méthode ne peut être utilisée que si la mer est calme, car s'il y a de la houle, il est difficile de repérer les flotteurs dans le creux des vagues. II est également indispensable de déterminer, avec grande précision, les positions de départ et d'arrivée des flotteurs; cette précision n'est réalisable qu'en vue des côtes. En pleine mer, on ne peut utiliser que les flotteurs dérivants qu'on laisse au gré des courants pendant des jours et même des mois et dont on détermine la position soit quand ils viennent s'échouer sur la côte, soit quand ils ont parcouru de nombreux kilomètres.

$$
\star^{\star} \star
$$

Nous avons passé rapidement en revue les divers courantomètres employés en océanographie en indiquant les avantages et les inconvénient de chacun d'eux.

A la fin de cette étude, on peut constater qu'il n'existe pas d'appareil parfait qui permette de mesurer les vitesses et les directions instantanées. II n'existe pas non plus d'appareil capable de déterminer les faibles vitesses.

Il serait souhaitable que les hydrodynamiciens, les océanographes et les électrotechniciens puissent associer leurs compétences pour mettre au point un bon appareil permettant de résoudre tous les problèmes qui se posent à l'océanographe.

Mais, à notre avis, il faut abandonner le principe du moulinet et s'orienter vers les appareils à fil chaud, ceux qui utilisent la résistance à l'avancement ou ceux qui sont basés sur les oscillations d'un pendule.

Il est impossible de concevoir un appareil universel pouvant travailler aussi bien en pleine mer que près des côtes, tant en surface que près du fond et adapté à tous les régimes de courant ; aussi il serait souhaitable d'étudier deux ou trois types d'appareils, basés peut-être sur des principes différents, mais bien adaptés au travail qu'on exigera d'eux. Ces appareils une fois mis au point, il serait souhaitable qu'ils puissent être universellement adoptés pour que toutes les mesures soient comparables. 